Catusse Myriam, «Tunisie et Égypte aux urnes ! Révolution ou restauration de la sociologie électorale ? », Confluences Méditerranée, 2012/3 (N82), p. 29-50.

0 DOI : 10.3917/come.082.0029. URL : http://www.cairn.info/revue-confluencesmediterranee-2012-3-page-29.htm

\title{
Tunisie et Egypte aux urnes !
}

2011, Révolution ou restauration de la sociologie électorale?

\author{
Myriam Catusse
}

\section{CNRS/IREMAM}

Ce papier se propose de réfléchir à la façon dont les scrutins de 2011 et 2012 en Tunisie et en Egypte bouleversent nos approches de l'acte électoral, à la fois sur ces deux terrains particuliers, mais également dans une visée comparatiste tenant compte des lectures privilégiées jusqu'alors. En contexte d'incertitude politique, il questionne ces élections «pas comme les autres » sous deux angles : il propose d'abord de ne pas se limiter aux lectures fonctionnalistes qui assignent au scrutin un rôle dans la reproduction des rapports de pouvoir ou au contraire dans le changement de régime (et la démocratisation); pour ensuite inviter sinon à la révolution du moins à une restauration, voire à une inauguration de la sociologie électorale sur ces terrains où, pour des raisons pratiques (les difficultés d'accès à l'information et la manipulation des résultats), mais aussi plus théoriques, celle-ci n'en est qu'à ses prémices. Il invite pour cela d'une part à envisager comment, malgré les ruptures introduites par la révolution, l'histoire électorale locale peut influencer aujourd'hui les candidatures, le vote et son herméneutique et d'autre part à questionner les innovations repérables dans ces scrutins plus libres et concurrentiels que les précédents : l'analyse des transformations de la représentation, de la restructuration des organisations partisanes, des métamorphoses du clientélisme et du leadership, mais aussi des comportements électoraux permet de questionner non seulement les héritages et décrochages entre l'ancien et le nouveau régime mais aussi entre la «politique de la rue » et celle «de l'urne », entre le «peuple en révolution » et le « corps électoral ».

En 1978 paraissait Aux urnes l'Afrique ${ }^{l}$, un ouvrage pionnier qui ouvrait alors des pistes de recherche comparatistes sur les scrutins dans les sociétés africaines sub-sahariennes. La même année, Des élections pas comme les autres ${ }^{2}$ proposait aussi de défricher des terrains

\footnotetext{
${ }^{1}$ D. Martin (dir.), 1978, Aux urnes l'Afrique. Elections et pouvoir en Afrique Noire, Paris, Pedone.

${ }^{2}$ G. Hermet, J. Linz, A. Rouquier, 1978, Des élections pas comme les autres, Paris, Presses de la FNSP ; G. Hermet, R. Rose, A. Rouquié (ed.), Elections Without Choice, London, Mc Millan, Publication de l'IPSA.
} 
jusqu'alors peu travaillés par la sociologie électorale, ceux du vote non-concurrentiel. Cette réflexion sur les prémices, les méthodes et les analyses de scrutins «sous contrôle » et «sans risque » pour les forces au pouvoir s'est approfondi au cours des décennies qui ont suivi. De nombreuses études de cas - beaucoup en Afrique sub-sahariennes - étaient mises en perspectives de réflexions plus comparatistes, innovantes alors en terme de décentrage des problématiques de la discipline. Les espoirs de «transition démocratique » au tournant des années 1990 intensifièrent ces recherches. Citons par exemple, pour leurs perspectives différentes, l'ouvrage dirigé par F. M. Howard ${ }^{3}$, dix ans plus tard le fameux numéro de Politique africaine, «Des élections comme les autres ${ }^{4}$ ou encore, pour s'en tenir à la littérature francophone, le Voter en Afrique sous la direction de P. Quantin ${ }^{5}$. Ces travaux témoignent de l'ancrage de la problématique électorale dans la science politique africaine et africaniste, sur plusieurs générations et avec des controverses scientifiques sur les manières de les aborder - des élections comme les autres ou pas comme les autres, vectrices de démocratisation ou ferments d'autoritarisme, etc.

Dans le monde arabe, si tant est que cette régionalisation de la sociologie politique ait un sens, force est de constater que l'intérêt pour l'objet élection, qui avait suscité pourtant des travaux de fond au lendemain des indépendances ${ }^{6}$, est vite retombé. Il a fallu attendre les années 1990, le réinvestissement du langage électoral des régimes comme de leurs partenaires étrangers derrière l'étendard galvaudé de la «transition démocratique », mais aussi la lecture du travail des africanistes, pour que les moments électoraux attirent à nouveau notre attention. Les scrutins algériens de 1990 et 1991, pourtant déterminants à bien des égards, furent singulièrement négligés ${ }^{7}$. Ce furent les travaux de J. F. Legrain sur les élections palestiniennes de l'autonomie en $1996^{8}$, et le collectif dirigé par S. Gamblin sur le scrutin de 1995 en Egypte $^{9}$ qui signalèrent le retour de l'élection sur l'agenda de la science politique dans le monde arabe. Un retour prudent et mesuré, aujourd'hui bousculé par le caractère historique de ces élections de 2011 où Tunisiens et Egyptiens purent aller aux urnes, dans des conditions $a$ priori très nouvelles.

\footnotetext{
${ }^{3}$ F. M. Howard (ed.), 1987, Elections in Inependant Africa, Boulder et Londres, Westview Press.

${ }^{4}$ R. Otayeck (dir.), 1998, « Des élections ‘comme les autres’ », Politique africaine, n69.

${ }^{5}$ P. Quantin (dir.), 2004, Voter en Afrique, Paris, Karthala.

${ }^{6}$ Je pense notamment aux articles dans Maghreb, l'Annuaire de l'Afrique du Nord ou la Revue française de science politique (sous les pseudonymes de O. Marais, H. Breton, P. Chambergeat ou J. et J. Aubin) et à la thèse de R. Leveau, 1976, Le fellah marocain défenseur du trône, Paris, Presses de la FNSP ; et plus largement aux premiers sommaires de l'Annuaire de l'Afrique du Nord, très sensiblement nourri de problématiques électorales.

${ }^{7}$ Avec des travaux néanmoins des travaux de fond comme la thèse sur les logiques partisanes de M. Aït Aoudia, 2008, L'apprentissage de la compétition pluripartisane en Algérie (1988-1992). Sociologie d'un changement de régime, doctorat de science politique, Université Paris 1, Panthéon-Sorbonne; et les travaux de S. Labat, 1995, Les islamistes algériens : des urnes au maquis, Paris, le Seuil.
}

${ }^{8}$ J. F. Legrain, 1999, Les Palestines au quotidien. Les élections de l'autonomie, janvier 1996, Beyrouth, Cahiers du CERMOC.

${ }^{9}$ S. Gamblin (dir.), 1997, Contours et Les élections législatives égyptiennes de 1995, Paris, l'Harmattan/CEDEJ. 
Ce papier se propose donc de réfléchir à la façon dont les scrutins de 2011 et 2012 en Tunisie et en Egypte bouleversent nos approches de l'acte électoral, à la fois sur ces deux terrains particuliers, mais également dans une visée comparatiste tenant compte des lectures privilégiées jusqu'alors. En quoi nos grilles de lectures sont-elles bouleversées par les soulèvements des Tunisiens et des Egyptiens ? Schématiquement, en Tunisie et en Egypte comme grosso modo dans le monde arabe les analyses des élections cherchèrent à répondre avant tout à deux questions principales : «des élections pourquoi faire ? ${ }^{10}$, une question qui appela principalement à l'analyse de la nature des régimes électoraux concernés et de leurs transformations sous contrôle ; et «des élections comme les autres ou pas comme les autres ? », une question qui, pour sa part, conduisit surtout à privilégier, comme nous y invitaient les africanistes de Politique africaine, une lecture anthropologique et «par le bas » des campagnes et de l'échange électoral, dans des contextes où les votes pouvaient être falsifiés et où l'enjeu du scrutin n'était pas le renouvellement des dirigeants du pays. Dans cette optique de nombreux travaux furent consacrés aux transformations des profils de la notabilité et du leadership ou aux formes multiples de l'échange électoral (et des logiques du clientélisme ou d'autres formes de solidarité), et plus généralement à une sociologie de la représentation. En revanche, la sociologie électorale, entendue comme l'étude du comportement des électeurs dans l'isoloir, resta largement ignorée de ces travaux : pour des raisons pratiques - le peu d'informations disponibles et fiables pour un traitement quantitatif des tendances électorales par exemple - ; et pour des raisons plus heuristiques - le soupçon partagé sur la falsification des résultats euphémisant l'intérêt de porter l'attention sur les chiffres officiels. Evidemment, dans le contexte révolutionnaire de 2011-2012, ces deux dernières interrogations phares sont singulièrement réactualisées mais demandent également à être dépassées ou reformulées. Quant aux freins à l'accès à l'information sur les votes, ils paraissent largement se lever (notamment en Tunisie) ${ }^{11}$, ouvrant de toutes nouvelles perspectives à la sociologie électorale.

Les élections pour l'Assemblée constitutionnelle tunisienne (le 23 oct. 2011), pour l'Assemblée législative égyptienne (du 28 nov. 2011 au 11 janvier 2012) ou pour la Présidence de la République égyptienne remportées le 24 juin 2012 par Mohammed Morsi, étaient marquées, pour la première fois dans l'histoire moderne de ces pays, du sceau de l'incertitude et de la compétition ouverte. A ce titre, elles dénotaient par rapport à celles qui leur avaient précédé et symbolisaient autant qu'elles introduisaient de sérieuses transformations dans ces deux régimes. Elles furent, de fait, observées très différemment des précédentes puisqu'elles appelèrent à l'intensification, voire dans le cas de la Tunisie quasiment à l'inauguration, d'une sociologie électorale : une approche qui tient compte non seulement des résultats des votes - tandis que jusqu'alors, il semblait convenu que les résultats ne comptaient peu, ou importaient moins que l'observation de ce qu'il se passait dans

\footnotetext{
${ }^{10}$ C'est le titre de deux papiers sur la Tunisie, séparés d'une décennie : V. Geisser, 2000, «Tunisie : des élections pour quoi faire? Enjeux et 'sens' du fait électoral de Bourguiba à Ben Ali », Maghreb/Machrek, Paris, la documentation française, $n^{\circ} 168$; É. Gobe et L. Chouikha, 2010, «Tunisie : des élections pour quoi faire ? Signification et portée des scrutins présidentiel et législatif de 2009 », Le Kiosque-Ceri, consultable en ligne sur http://www.ceri-sciences-po.org/

${ }^{11}$ Ainsi l'illustre la contribution de A. Gana dans ce volume.
} 
la campagne ${ }^{12}$-, mais encore du comportement des électeurs en lien avec leurs caractéristiques sociales, avec les processus qui peuvent générer des identifications et des préférences politiques. Encore peu documentés sur les sous-sols sociopolitiques de la révolte et curieux de mieux connaître ceux qui firent, «anonymes » dit-on, la révolution, les observateurs découvrent aussi enfin progressivement les électeurs et les électrices tunisiensnes et égyptiens-nes. Ceux qui allèrent aux urnes; et dans une moindre mesure, ceux qui ne s'y rendirent pas.

Dans ce contexte, ces derniers scrutins appellent peut être moins à une révolution de fond en comble de la sociologie politique, qu'à une «restauration» critique et sous contrôle de certains outils et grilles d'analyse, en rupture ou dans le prolongement des jalons naissant de la sociologie électorale sur ces terrains ${ }^{13}$. Par intuition, on peut faire l'hypothèse que certains des processus attribués aux Révolutions des 15 janvier et 25 janvier peuvent leur être antérieurs ou encore que certains des rapports de force et comportements politiques dans ces scrutins exceptionnels gagnent à être expliqués dans une histoire plus longue que celle qui débuta par l'immolation de Mohammed Bouazizi. Il s'agit donc d'une part de questionner à l'aune des travaux précédents et des enquêtes plus récentes, les accroches et décrochages de la politique des urnes à celle la rue, celle des manifestants et insurgés qui occupèrent la place Tahrir du Caire ou celle de la Casbah à Tunis ${ }^{14}$; d'autre part et, a contrario, d'explorer ce qui peut apparaître comme inédit, produit ou catalysé par le mouvement social, à commencer par le degré important d'incertitude et l'émergence d'un corps électoral. Au-delà du fait révolutionnaire et de l'incontestable caractère inédit de ces élections, il s'agit donc aussi de réfléchir aux conditions de renouvellement de nos propres grilles de lecture.

\section{Des élections pour quoi faire?}

Une première question régulièrement posée au sujet des scrutins de 2011 et 2012 en Tunisie et en Egypte concernait la «fonction» de ces élections dans le processus de changement de régime qui s'accélère. Cette interrogation de théorie politique n'est pas vraiment nouvelle quand bien même ici elle prend des accents tout inédits et bien légitimes : prolonge-t-elle la révolution ou l'interrompt-elle ? Signe-t-elle l'avènement de régimes démocratiques ou consacre-t-elle de nouvelles formes de domination ? C'est une question qui a accompagné la

\footnotetext{
12 Certains comme S. Soliman montrèrent néanmoins quelques intérêts de travailler ces résultats, en connaissance de cause, pour montrer par exemple que la participation électorale s'avérait inversement proportionnelle du niveau de vie lors des derniers scrutins égyptiens, engageant par là à travailler sur l'échange électoral et la notion de «service» (khidma) dans le travail de représentation : S. Soliman, 2007, La participation politique dans les élections législatives de 2005 (en arabe), Le Caire, The Egyptian Association for Community Participation Enhancement.

${ }^{13}$ A l'instar de ceux posés par S. Ben Néfissa et A. al-Dîn Arafat, et démocratie dans l'Egypte contemporaine, Paris, Karthala.

${ }^{14}$ Sur d'autres terrains, voir le récent S. Luck, S. Dechezelles (dir.), 2011, Voix de la rue ou voies des urnes?, Rennes, PUR.
} 
reprise des analyses électorales dans le monde arabe depuis les années 1990, à la faveur de l'observation de scrutins présentés comme «exceptionnels »- à l'instar des législatives de 1995 en Egypte présentés alors par le régime comme plus ouvertes que les précédentes ${ }^{15}$; ou à l'instar des communales de 1998 au Liban qui reprenaient un cycle électoral local interrompu pendant 30 ans, du fait de la guerre civile ${ }^{16}$, , voire potentiellement «fondateurs » - d'un régime qui se met en place dans le cas des premières élections dans les territoires occupés de $1996^{17}$, ou de nouveaux régimes électoraux, plus ouverts, comme ce fut le cas des législatives de 1997 au Maroc, qui présidèrent à la formation d'un "gouvernement d'alternance » en $1998^{18}$. Néanmoins, si les spécialistes de l'Afrique sub-saharienne voisine s'engagent à cette époque résolument dans l'étude des scrutins comme lieux privilégiés de potentielles «transitions démocratiques», dans une sorte de nouveau «triomphe de l'élection» (justifié par la nature même des revendications électoralistes qui animent de nombreuses sociétés subsahariennes des années $1990^{19}$ ), sur les terrains arabes de l'élection au contraire, en Egypte et en Tunisie notamment, la perspective s'inverse : ces scrutins sont volontiers observés comme des outils de discipline, aux mains de régimes peu démocrates en place depuis plusieurs décennies ${ }^{20}:$ «refusant de les réduire à un trop plein (de fraude et de violence) ou à un manque (de transparence et de liberté), ces élections sont envisagées comme un mécanisme central dans un régime autoritaire contraint à se réformer $\gg^{21}$. L'accent est mis sur les procédures de façade (certains s'aventurent à parler de démocratie formelle (dimucratiyya shakliyya)), sur la «fiction pluraliste ${ }^{22}$ qui s'ancre sans que personne ne soit réellement dupe. "S'il y avait un quelconque intérêt à rendre compte des élections, ce n'est pas de ses enjeux électoraux eux-mêmes (les résultats sont connus à l'avance) que dans les cérémonies et les rituels politico-électoraux relevant à la fois de la permanence et de l'innovation » indiquent E. Gobe et V. Geisser au sujet des présidentielles tunisiennes de $2004^{23}$. Selon les cas, l'analyse se porte donc sur les formes de mobilisation « par en haut », de cooptations, d'élimination de potentiels adversaires ou de fragmentation du

\footnotetext{
${ }^{15}$ S. Gamblin (dir.), 1997, op. cit.

${ }^{16}$ A. Favier (dir.), 2002, Municipalités et pouvoirs locaux au Liban, Beyrouth, Cahiers du CERMOC.

17 J. F. Legrain, 1999, op. cit.

${ }^{18}$ J. Ph. Bras (dir.), 2000, « Dossier : Élections et représentation au Maghreb », Maghreb-Machrek, $\mathrm{n}^{\circ}$ 168, avriljuin

19 M. Bratton, N. Van de Walle (ed.), 1997, Democratic Experiment in Africa. Regime Transitions in Comparative perspective, New York, Cambridge University Press. Voir aussi P. Quantin, 1993, "Afrique”, in P. Perrineau et D. Reynié (dir.), Dictionnaire du vote, Paris, PUF, pp. 22-26.

${ }^{20}$ J. Schwedler, L. Chomiak, 2006, «And the Winner is... Authoritarian Elections in the Arab World », Middle East Report, vol. 36, n²38, p. 12-19.

${ }^{21}$ F. Kohstall, F. Vairel, 2011, «Préface », in F. Kohstall, F. Vairel (dir.), «Fabrique des élections », Egypte. Monde arabe, $\mathrm{n}^{\circ} 7,3^{\mathrm{ème}}$ série, $\mathrm{p}$. XI.

${ }^{22}$ O. Lamloum , B. Ravenel, 2000, «Tunisie : la fiction pluraliste », Confluences méditerranéennes, $\mathrm{n}^{\circ} 32$, hivers 1999-2000, pp. 173-182.

${ }^{23}$ V. Geisser, É. Gobe, 2005, «Tunisie : consolidation démocratique et processus électoraux », Année du Maghreb, CNRS éditions, p. 328.
} 
paysage partisan ${ }^{24}$, de fraudes et violences politiques, d'achat de votes, ou encore de promotion d'élites loyales et redevables au pouvoir central, etc. ${ }^{25}$ : sous cet angle, le réinvestissement de l'ingénierie électorale par les régimes en place sert à «asseoir les dispositifs de contrôle de la société, les fidèles étant récompensés, les indécis mobilisés et les récalcitrants réprimés $»^{26}$.

Si l'élection est l'enfant chéri de la démocratie, conquête et objet de désir des mouvements d'émancipation des peuples, elle ne fait donc pas pour autant la démocratie. Il n'a pas fallu attendre ces scrutins des années 1990, ni les incertitudes présentes sur le devenir des régimes égyptiens et tunisiens, ni même l'adage de mai 1968 emprunté à J. P. Sartre, « élections, piège à cons », pour montrer à quel point l'histoire sociale et politique des institutions de la démocratie représentative sont tout à la fois des outils d'expression de la vox populi mais aussi de manipulations, d'exercices de cens et d'aristocratismes ${ }^{27}$, de formes variées de domination et d'illusions procédurales ${ }^{28}$. Elles ont à plusieurs reprises fermé le cycle des mobilisations, servi de «puissant antidote aux passions et émotions révolutionnaires, (...) s'étant imposée[s] (finalement) comme un mode de régulation des conflits et de neutralisation du pouvoir de surprise que détenait notamment le peuple émeutier ${ }^{29}$. Sur les terrains africains d'ailleurs que nous évoquions plus haut, force est de constater que si la récente alternance sénégalaise a pu témoigner de l'ancrage progressif de pratiques concurrentielles, dans la plupart des cas, le bilan de vingt ans d'expériences électorales pluripartites est mitigé. Elles ne se sont accompagnées «ni d'une extension des droits et libertés politiques, ni d'une alternance partisane, ni même de l'émergence d'un système partisan stable $»^{30}$. Les exemples sont nombreux d'ailleurs où le scrutin s'apparente moins à une conquête de pouvoir qu'à sa confiscation, et pas seulement à celui des révolutionnaires ${ }^{31}$. A ce titre, la poursuite par les autorités des leaders et organisations du mouvement du 20 février marocain pour avoir appelé au boycott du référendum organisé le $1^{\text {er }}$ juillet 2011 est

${ }^{24}$ E. Lust-Okar, 2004, «Divided They Rule: the Management and Manipulation of Political Opposition », Comparative Politics, vol. 36, n², pp. 159-179.

25 Pour une analyse critique de ces approches: M. Bennani Chraïbi, 2004, «Introduction. Représenter et mobiliser dans l'élection législative au Maroc », in M. Bennani Chraïbi, M. Catusse, J. C. Santucci (dir.), Scènes et coulisses de l'élection au Maroc, Paris, Karthala, pp. 15-54.

${ }^{26}$ V. Geisser, É. Gobe, 2005, article cité, p. 327.

${ }^{27}$ D. Gaxie, 1978, Le cens caché. Inégalités culturelles et ségrégations politiques, Paris, Seuil.

${ }^{28}$ B. Manin, 1996, Les principes du gouvernement représentatif, Paris, Flammarion ; P. Rosanvallon, 1998, Le peuple introuvable. Histoire de la représentation démocratique, Paris, Gallimard.

${ }^{29}$ O. Dabène, M. Hastings, 2007, «Introduction », in O. Dabène, M. Hastings (dir.), La surprise électorale. Paradoxes du suffrage universel, Paris, Karthala/CSPC, p. 6.

${ }^{30}$ P. Quantin, 2009, «Politics et policies vont dans un bateau : que reste-t-il à la classification des régimes politiques africains ? », in M. Camau, G. Massardier (dir.), Démocraties et autoritarismes. Fragmentation et hybridation des régimes, Paris, Karthala, p. 201.

${ }^{31}$ Le président de la Haute instance pour la réalisation des objectifs de la révolution, de la réforme politique et de la transition démocratique en Tunisie, rappelle ainsi dans une interview la fameuse formule Napoléonienne : «dans toute révolution, il y a ceux qui la font et ceux qui en profitent ». Interview à Shems FM, 03/11/2011 : sources: http://www.shemsfm.net/fr/actualite/actualites shems-news/y-ben-achour-la-revolution-une-proie-pourcertains-profiteurs $/ 12$ 
édifiante $^{32}$; et auparavant, plus frappantes encore furent ce que l'on appela pudiquement «l'interruption du processus électoral » dans l'Algérie des années 1990/1991 suivies de sombres années de guerre civiles, ou encore la suspension de l'aide internationale aux Territoires occupés face au verdict des urnes au profit du Hamas aux élections de 2006, suivie de la proclamation de l'Etat d'urgence et de la partition de facto de Gaza et de la Cisjordanie $^{33}$.

Ainsi comprend-on tout l'intérêt de se libérer en partie, y compris aujourd'hui où le devenir des régimes tunisiens et égyptiens reste en suspens, de questions purement fonctionnalistes pour observer ce qui se joue dans les élections en dessous du seuil de la démocratisation ${ }^{34}$. C'est finalement le parti pris de beaucoup de ces travaux qui ne considèrent la « réforme autoritaire » que comme la toile de fond d'analyse des élections plus que comme son alpha et oméga. Leurs analyses stimulantes, en décalage profond tant avec les approches transitologiques qu'avec l'idée selon laquelle les élections dans ces contextes seraient de «mauvais objets scientifiques $»^{35}$, montrent comment au-delà du constat d'évidence que "les élections se suivent... et se ressemblent ${ }^{36}$, malgré d'éventuelles reprises en main ou au contraire «dérapages démocratiques ${ }^{37}$, le changement d'échelle d'observation, jusqu'au «ras du terrain ${ }^{38}$, permet de mettre en évidence des anomalies, des histoires singulières et non généralisables, des nouveautés, voire des transformations politiques. On le lit à leurs intitulés : «Contours et détours du politique » en Egypte, «scènes et coulisses de l'élection » au Maroc, il s'agit pour les observateurs de débusquer la nouveauté («qu'est-ce qui a changé par rapport aux élections précédentes ? ${ }^{39}$ ), de découvrir des éléments originaux et peu

\footnotetext{
${ }^{32}$ Plus précisément, les autorités marocaines démentent que l'appel au boycott ait été l'objet de poursuite (1(objet du délit étant la diffusion de tracts contraires à la loi). Néanmoins, organisations des droits de l'homme, organisations politiques et organismes internationaux comme Human Rights Watch s'indignent de la procédure : sources : $\quad$ http://www.hrw.org/fr/news/2012/02/19/maroc-les-autorit-s-devraient-abandonner-les-poursuitescontre-des-militants-ayant-a

33 J. F. Legrain, 2006, «L'impasse politique et institutionnelle palestinienne », Critique internationale, juilseptembre, doc. additionnels numériques.

${ }^{34}$ L'expression est de S. Heydemann envisageant « la possibilité que des changements importants fussent, dans ces régions, en cours de maturation en dessous du seuil où l'autoritarisme s'effondre, celle que des formes nouvelles, hybrides, d'autoritarisme y fussent en gestation», in S. Heydemann, 2002, «La question de la démocratie dans les travaux sur le monde arabe », Critique internationale, n 17 , oct., p. 58.
}

${ }^{35}$ R. Otayek, 1998, «Introduction au thème. Les élections en Afrique sont-elles un objet scientifique pertinent », Politique africaine, n 69 , mars, p. 3-11.

${ }^{36}$ O. Lamloum, B. Ravenel, 2000, op. cit. p. 174. Voir aussi I. Farag, 2006, « De Moubarak à Moubarak. L’élection présidentielle de 2005 en Egypte », Critique internationale, n³3, 2006.

37 « Les normes démocratiques produisent (...) des effets du fait même de leur adoption, indépendamment de ce que pensent leurs importateurs. Le changement institutionnel va entrainer un changement dans l'attitude des acteurs et susciter de nouveaux engagements. L'adoption d'institutions démocratiques crée, à plus ou moins court terme, des comportements favorables aux démocraties », J. N. Ferrié, N. Bernard Maugiron, 2008, « Les architectures constitutionnelles: introduction », Egypte/Monde arabe, $3^{\text {ème }}$ série, Les architectures constitutionnelles des régimes politiques arabes, mis en ligne le 08 juillet 2008.

${ }^{38}$ L. Zaki, 2009, "Pour une analyse localisée des élections marocaines », in L. Zaki (dir.), Terrains de campagne au Maroc. Les élections législatives de 2007, Paris, Karthala/IRMC, p. 17.

${ }^{39}$ A. Roussillon, 2011, « Les coptes à la marge », in F. Kohstall, F. Vairel, op. cit., p. 95. 
visibles qui ne s'expliquent pas à l'unique aune des mécanismes de l'autoritarisme, dans les détails d'une affaire, les dispositifs de la loi et ses usages, la trajectoire d'un homme ou le déroulé d'une campagne ou encore l'expression de multiples solidarités. L'un des intérêts, et non des moindres - au vu des soulèvements de 2011, de ces recherches sur des élections aux résultats attendus fut donc de rendre compte de dynamiques plurielles et variées, parfois subreptices et souvent localisées, dans ces sociétés politiques parfois trop caricaturalement dépeintes comme de «belles endormies », soumises au joug de pouvoirs répressifs et non pluralistes, voire «achetées » en échange de la redistribution arbitraire (et peu généreuse pourtant) de rentes publiques.

Cette prudence quant à l'articulation entre élection et démocratisation qui a caractérisé la réflexion sur les terrains arabes de ces deux dernières décennies reste donc largement de mise dans les travaux plus récents sur les scrutins de 2011 et 2012. De même que l'héritage des questions posées sur les logiques de la représentation, en contexte d'élections non concurrentielles, continue de peser sur les grilles de lecture des votes post-révolutionnaires, pour montrer notamment que ces derniers ne s'expriment pas ex-nihilo mais bel et bien dans une histoire complexe de l'échange électoral que la révolte et le mouvement social ont infléchit sensiblement sans l'interrompre ex-abrupto.

\section{Les enjeux d'une (nouvelle) sociologie électorale}

Quels legs de la sociologie politique pour comprendre les métamorphoses actuelles de la représentation dans la Tunisie et l'Egypte de la révolution? La sociologie politique a fait varier les échelles, « en passant de la sphère institutionnelle (le jeu politique) au champ social (le jeu social), de la macro-situation (jeux politique et social) à la micro-situation (trajectoires sociales et profils d'acteurs) $»^{40}$. Partant, elle s'est essentiellement organisée jusqu'en 2011, comme l'indique le tableau que M. Bennani-Chraïbi brossait des approches privilégiées jusqu'alors au $\operatorname{Maroc}^{41}$ (à peu de choses près, les mêmes tendances se repèrent en Tunisie et en Egypte) autour d'approches technicistes ou d'analyse des modes de (re)production des élites. Ces deux types d'approches peuvent d'être remobilisées dans l'observation des scrutins exceptionnels de 2011/2012, pour ce qu'elles peuvent nous dire des innovations et des transmissions par delà la révolution.

\section{Le droit à l'élection}

Certains apportèrent un intérêt tout particulier aux dimensions procédurales, légales, aux « règles du jeu » régissant le moment électoral, y compris dans ces sociétés d'arbitraire (l'Etat

\footnotetext{
${ }^{40}$ M. Hachemaoui, 2003, «La représentation politique en Algérie entre médiation clientélaire et prédation (1997-2002), RFSP, vol. 53, nº fév., p. 35.

${ }^{41}$ M. Bennani-Chraïbi, 2004, «Introduction. Représenter et mobiliser... », article cité.
} 
d'urgence est proclamé en 1981 en Egypte pour n'être levé qu'en mai 2012). C'est notamment l'option suivie par F. Kohstall et F. Vairel dans leur récent collectif sur les législatives et présidentielles de 2005 en Egypte, qui mettent l'accent sur "l'instabilité des règles $d u$ jeu » et les dilemmes pratiques que cela induit pour les acteurs du scrutin, depuis les candidats jusqu'aux électeurs en passant par les garants de la régularité des procédures ${ }^{42}$. A côté de l'analyse d'une «transposition en contexte autoritaire de mécanismes inhérents aux régimes démocratiques ${ }^{43}$ à laquelle engagent ces auteurs - une problématique finalement qui rejoint les analyses en termes d'hybridation des régimes politiques ${ }^{44}$ - retenons que ces approches ont mis en évidence - en Egypte plus qu'en Tunisie - des formes complexes de judiciarisation du politique - autorisant le judiciaire à se faire juge du politique -, y malgré le faible Etat de droit. Loin de se présenter comme des logiques de dépolitisation de l'arène politique, elles dénotent au contraire une régularisation du recours à l'arme du droit dans le rapport de force politique, de la part de professionnels et de profane du juridique. Ainsi, le rôle des juges dans les processus électoraux égyptiens actuels sont à saisir dans une histoire longue et conflictuelle finement analysée sur plus d'une décennie par N. Bernard Maugiron ${ }^{45}$. C'est dans ce sens que l'on pourrait aussi analyser aujourd'hui la place qu'occupe dans le processus électoral égyptien la Haute cour constitutionnelle ${ }^{46}$, et plus largement le poids des avocats et des juges. Le club des juges en Egypte, comme le barreau tunisien qui figurent parmi les rares contre-pouvoirs aux régimes de Moubarak et de Ben $\mathrm{Ali}^{47}$, jouent désormais dans la régulation de la compétition politique un rôle parfois déterminant, plus ou moins inédit selon que l'on s'intéresse à la Tunisie ou à l'Egypte : tant pour leurs forces de pression corporatistes ou moins sectorisées, que pour la façon dont ils contribuent à faire des points de procédure et des mécanismes juridiques des éléments centraux dans le changement de régime $^{48}$. Le travail de la Haute instance pour la réalisation des objectifs de la révolution en

\footnotetext{
${ }^{42}$ Voir aussi, dans la même optique, E. Lust-Okar, A. Ahmad Jamal, 2002, «Rulers and Rules. Reassessing the Influence of Regime Type on Electoral Law formation », Comparative Political Studies, vol. 35, $\mathrm{n}^{\circ} 3$, April, pp. 337-366.

${ }^{43}$ F. Kohsatll, F. Vairel, 2011, «Les élections législatives et présidentielles en Egypte de 2005 à 2010 », in F. Kohstall, F. Vairel, «Fabrique... », op. cit., p. 6.

${ }^{44}$ M. Camau, G. Massardier, Démocraties et autoritarismes..., op. cit.

${ }^{45}$ N. Bernard Maugiron, 2009, Judges and Political Reform in Egypt, Le Caire, Presses de l'Université américaine du Caire ; «Les juges et la supervision des élections de 2005 », in F. Kohstall, F. Vairel (dir.), «Fabrique des élections », Egypte-Monde arabe, pp. 129-156.

${ }^{46}$ Dont le rôle dans la régularisation des opérations électorales dans des entre deux procéduraux et plus substantiels a été démontré par E. Kienle, 2006, «L'autoritarisme en chantier: l'Egypte entre dispositifs d'ouvertures, tendances libéralisatrices et restrictions de rattrapage », in J. N. Ferrié, J. C. Santucci (dir.), Dispositifs de démocratisation et dispositifs autoritaires en Afrique du Nord, Paris, CNRS édition, pp. 107-120.

${ }^{47}$ Voir sur ce point les travaux de É. Gobe (2012), dans le cadre d'une Habilitation à diriger des recherches, État, Barreaux et avocats en Tunisie de la colonisation à la révolution (1883-2011). Contribution à une sociologie politique des professions.

${ }^{48}$ Voir à ce sujet la contribution de M. Lieckefett dans ce volume sur le travail de la Haute instance pour la réalisation des objectifs de la révolution, de la réforme politique et de la transition démocratique en Tunisie du 15 mars au 13 octobre 2011. A titre de comparaison: J. Heurtaux, 2006, « Le changement de régime au prisme des usages politiques du droit. 1989 et l'entrouverture de la compétition politique en Pologne », in S. Kott, M. Mespoulet, A. Roger (dir.), Le post-communisme dans l'histoire, Bruxelles, Presses Universitaires de Bruxelles, pp. 37-50.
} 
Tunisie (la rédaction d'une nouvelle loi électorale et l'élection de l'Instance supérieure indépendante pour les élections (Isie) dont elle défini l'organisation), aussi inédit et exceptionnel soit-il, gagne ainsi à être mis en perspective avec l'inflation au cours de la décennie précédente d'instances et de dispositifs d'organisation et de contrôle des scrutins ${ }^{49}$.

Dans le chapitre des questions soulevées par les techniques du vote et leurs encadrements procéduraux, peu d'observateurs sur nos terrains se sont, en revanche, jusqu'à présent investis dans une histoire sociale et matérielle du vote à la façon en France de O. Ihl, Y. Deloye ou A. Garrigou $^{50}$. S. Ben Nefissa et A. al Dîn Arafat sont sans doute ceux qui ont poussé le plus loin ces pistes dans le cas de l'Egypte pour montrer comment de scrutins en scrutins, les pratiques électorale et leurs normes se sont constituées «progressivement à partir des pratiques et de l'opération de classement et de (dé)légitimation qui les a affectés ${ }^{51}$, y compris les pratiques de fraude et de clientélismes qui jonchent l'histoire de l'ancrage de la compétition électorale et des systèmes représentatifs sur nos terrains ${ }^{52}$ mais ailleurs aussi ${ }^{53}$. Les observations de M. Béchir Ayari dans les bureaux de vote du Grand Tunis en octobre $2011^{54}$ trouvent échos dans ces travaux, en montrant comment, par-delà les données totalement inédites de l'élection de 2011, l'individualisation du vote, bien que souhaitée par les observateurs internationaux dépêchés sur place pour ces premières élections libres, ne va pas nécessairement de soi et doit au contraire s'analyser dans un long processus de façonnage et de fabrication du corps électoral : à coup de réformes des modes de scrutin, de (re)découpage des circonscriptions, d'introduction de règles spécifiques à l'égard de certaines catégories d'électeurs ou de candidats $^{55}$, de distribution de cartes électorales, etc. En tout état de cause, la problématique de l'articulation entre les dimensions procédurales et substantielles de la démocratie reste largement ouverte dans ce temps insurrectionnel, et méritera certainement de plus fins développements pour explorer la façon dont les techniques du vote contribuent à encadrer et contenir celui-ci autant qu'à permettre l'expression libre et individuelle des préférences politiques des citoyens tunisiens et égyptiens, voire, fait nouveau dans ces changements de régimes, à garantir les conditions de l'expression d'intérêts divergents (ce que le rapport de force dans la rue permet plus difficilement, sinon au risque de l'usage de la force).

\footnotetext{
${ }^{49}$ F. Vairel, 2009, «Observer les élections : action publique et contrainte sur la " société civile" », in L. Zaki (dir.), Terrains de campagne..., op. cit., pp. 217-237.

50 A. Garrigou, 2002, Histoire sociale du suffrage universel en France. 1848-2000, Paris, Seuil ; O. Ihl, Y. Deloye, 2008, L'acte de vote, Paris, presses de Sciences-Po.

${ }^{51}$ S. Ben Néfissa, A. al Dîn Aafat, 2005, Vote et démocratie..., op. cit., p. 14.

${ }^{52}$ Sur des terrains égyptiens, voir M. Vannetzel, 2008, « Les voies silencieuses de la contestation : les Frères musulmans égyptiens entre clientélisme et citoyenneté alternative », raisons politiques, $\mathrm{n}^{\circ} 29$, pp. 23-37. T. Aclimandos, «Splendeurs et misères du clientélisme», in F. Kohstall, F. Vairel (dir.), «Fabrique des élections... », op. cit., pp. 197-217. Et en Algérie: M. Hachemaoui, 2003, «La représentation politique en Algérie... », article cité.

${ }^{53}$ J. L. Briquet, F. Sawicki (dir.), 1998, Le clientélisme politique dans les sociétés modernes, Paris, PUF.

${ }^{54}$ M. Béchir Ayari, 2011, «Petite ethnographie des bureaux de vote du Grand Tunis », intervention à la Journée d'étude «Processus électoraux, révolutions et transitions en Egypte et en Tunisie », Tunis, IRMC/IRD/IREMAM.

55 Par exemple, l'introduction de quota de femmes et de jeune (dans le cas de la loi électorale marocaine), voire l'obligation de parité de genre dans les listes électorales (dans le cas de la loi électorale Tunisienne de 2011).
} 


\section{Métamorphoses de la représentation?}

C'est aussi à l'aune d'une sociologie des élus et « représentants » du peuple, que l'analyse des scrutins de 2011 et 2012 peut mettre en relief les effets de rupture introduits par le mouvement social ou au contraire des processus moins directement affectés par la révolution. Les scrutins précédents ont, de fait, été des lieux privilégiés d'observation sinon des comportements électoraux, du moins de la formation et de la transformation de leaderships. C'est à l'échelle locale que les analyses se sont faites les plus fines pour rendre compte des logiques de la représentation, et de leurs mise à l'épreuve électorale : les députés égyptiens «ne sont pas seulement des représentants d'une caste de détenteurs et bénéficiaires du pouvoir, cooptés par ce même pouvoir, mais encore ceux de groupes qui s'imposent comme dominants dans le système social ${ }^{56}$. Le même constat est partagé par beaucoup de ceux qui cherchent à rendre compte des transformations des sociétés politiques locales arabes ${ }^{57}$.

Une première tendance mise en exergue ces dernières années, notamment sur les terrains égyptiens (et marocains), concerne la transformation des figures de la notabilité au profit de nouvelles aristocraties et de cens inscrits dans ce qu'en Afrique Sub-saharienne J. F. Bayard appela la «politique du ventre $»^{58}$. Les formes de l'interventionnisme administratif dans le scrutin ont évolué au gré des réformes procédurales des règles électorales, et dans un contexte de libéralisation économique sous contrôle: la manipulation des votes passe moins par le trucage et le bourrage des urnes (même si ces pratiques demeurent) que par l'extension de la marchandisation du bulletin. Dans certains cas, les observateurs pointent l'achat pur et simple des voix, des pratiques de «prédation » qui troublent d'ailleurs les formes plus routinières et complexes de clientélisme qui ne s'arrêtent pas au seul moment électoral, à tel point que l'on peut considérer que «l'achat de voix n'est pas le stade suprême du clientélisme, mais le signe le plus sûr de sa déréliction ${ }^{59}$. Dans d'autres cas, c'est la politique du service (khidma) qui est mise en avant: l'échange de protection (de divers filets de sécurité sociale notamment, dans des contextes où l'Etat de providence est en lambeaux, en Tunisie certainement moins qu'en Egypte) ou de services collectifs contre le soutien électoral des clients envers le patron. Ainsi, les scènes politiques se sont de fait «embourgeoisées » à la faveur de l'ajustement structurel, avec de nouvelles trajectoires de l'accumulation économique et... politique ; elles accueillirent volontiers des outsiders de la politique, hommes d'affaires et patrons, qui se présentèrent plus qu'auparavant aux rendez-vous électoraux et se firent mieux élire que d'autres catégories socioprofessionnelles, pour des raisons variées qui relèvent autant du déplacement des échelles du prestige et de la réussite derrière le slogan de la libéralisation économique que de la capacité matérielle à mener campagne et à fidéliser des électeurs. Dans

\footnotetext{
${ }^{56}$ É. Longuenesse, 1997, « Logiques d'appartenance et dynamiques électorales dans une banlieue ouvrière : le cas de la circonscription 23 à Helwân », in S. Gamblin (dir.), Contours et détours..., op.cit.

${ }^{57}$ M. Hachemaoui, 2003, « La représentation politique... », article cité, p. 35.

${ }^{58}$ J. F. Bayard, 2006, L'Etat en Afrique : la politique du ventre, Paris, Fayard.

${ }^{59}$ T. Aclimandos, 2011, « Splendeurs et misères du clientélisme », article cité, p. 207.
} 
la région, ces travaux concernent essentiellement l'Egypte et le Maroc ${ }^{60}$, moins la Tunisie où la transformation des profils de la notabilité reste moins documentée sur les périodes les plus récentes ${ }^{61}$. On peut le regretter tant les clivages sociaux y semblent avoir été l'un des moteurs du mouvement social. Depuis la révolte jusqu'aux discussions autour des nouvelles règles du jeu politique, les solidarités ou divergences régionalistes mais aussi de classes ont nourri les mobilisations et dessiné en creux ou en plein les recompositions de leaderships dont il faudra certainement faire une histoire sociale par delà la révolution, afin de saisir en quoi cet évènement a-t-il engendré, confirmé ou infirmé, par les urnes plus que dans la rue, l'émergence de «néo-notables $»^{62}$.

En Egypte, ces travaux donnèrent lieu à de fins travaux sur le clientélisme, que l'on ne reprendra pas ici, sinon pour mettre en avant les logiques plurielles de ces relations politiques, de l'usage matériel de l'argent jusqu'à celles du «clientélisme électoral concurrentiel », qui rééquilibre les rapports de force politique au bénéfice des électeurs. Cette expression, empruntée aux travaux de C. Goirand sur la politique dans les favelas brésiliennes ${ }^{63}$, fut aussi utilisée au sujet des pratiques électoralistes du PJD marocain ${ }^{64}$ qui trouve des échos très stimulants dans les modalités de mobilisation des Frères musulmans en Egypte décrites par M. Vannetze ${ }^{65}$. Alors que le contexte de concurrence politique s'exacerbe au gré des scrutins successifs, avec un éclatement du paysage partisan (et le renoncement formel au monopartisme), l'offre électorale s'est élargie, quand bien même l'élection reste sous contrôle étroite. Face à cela, les liens entre patrons et clients sont déstabilisés, et pas seulement au détriment du corps électoral. M. Vannetzel montre comment face aux violences et dominations, celui-ci se «fait»dans ces scrutins, à côté de pratiques d'escapisme (ne pas oublier que malgré les corrections de l'administration, les taux de participation dans les années 2000 sont demeurés faibles). Les électeurs peuvent ainsi jouer du moment électoral pour faire entendre leur voix, du moins exprimer leurs doléances aux candidats de leurs circonscription, voire promouvoir la «participation politique des oubliés » par le biais du relais que leur offre en l'occurrence les Frères musulmans, un relais fait de services présentés comme dépersonnalisés, tissés au quotidien et non en lien direct avec l'élection. Ces travaux appuyés sur des enquêtes localisées qui ne permettent pas nécessairement la généralisation insistent sur le fait que «pour répondre aux attentes des électeurs et asseoir leur autorité, les nouvelles élites politiques ne peuvent se contenter de jouer un rôle de courroie de

\footnotetext{
${ }^{60}$ M. Catusse, 2008, Le Temps des entrepreneurs. Politique et transformations du capitalisme au Maroc, Paris, Maisonneuve et Larose/IRMC; M. Bennani Chraïbi, 2008, «'Hommes d'affaires' versus 'profs de fac'. La notabilisation parlementaire d'un parti de militants au Maroc », Revue internationale de politique comparée, Vol. 15, n², pp. 205-219.

${ }^{61}$ En revanche l'histoire sociale de la notabilité en Tunisie a fait l'objet de travaux documentés : voir A. Hénia (dir.), 2006, Etre notable au Maghreb : Dynamique des configurations notabiliaires, Paris, Maisonneuve \& Larose/IRMC.

${ }^{62}$ Mutatis mutandis, à l'image de ce qu'observa J. F. Legrain dans les territoires occupés à l'occasion de l'organisation en 1996 des premières élections de «l'autonomie » : J. F. Legrain, 1999, Les Palestines ..., op. cit.

${ }^{63}$ C. Goirand, 2000, La politique des favelas, Paris, Karthala-CERI.

${ }^{64}$ M. Catusse, L. Zaki, 2009, « Gestion communale et clientélisme moral au Maroc: les politiques du Parti de la justice et du développement $»$, Critique internationale, janvier, vol ${ }^{\circ} 42, \mathrm{n}^{\circ} 1, \mathrm{pp} .73-91$.

${ }^{65}$ M. Vannetzel, 2008, « Les voies silencieuses de la contestation... », article cité.
} 
transmission des directives du centre politique (qui leur est pourtant traditionnellement alloué par le régime autoritaire) : elles sont souvent obligées d'abandonner leur posture de courtiers privilégiés pour adopter celles de porte-parole et de défenseurs des intérêts de leurs électeurs ${ }^{66}$.

En somme, ces plongées dans les transactions entre candidats, partis et électeurs qui a occupé l'attention des observateurs en Egypte (comme au Maroc ou en Algérie principalement mais aussi au Liban dans de récents travaux ${ }^{67}$ ) invitent à lire les logiques des scrutins de 2011 à l'aune des questions qu'elles posent : comment se réorganisent les relations de clientèle au niveau local alors que l'offre politique s'avère encore plus importante dans ces scrutins de la révolution (en Tunisie, plus d'une centaines de partis sont en lice en octobre 2011)?

Comment en Egypte, la «politique de services» des Frères musulmans, observée sur plusieurs décennies va-t-elle évoluer à la faveur des victoires électorales du parti, sa conquête de postes de députés, mais aussi de l'appareil d'Etat ${ }^{68}$ ? Comment expliquer parallèlement les premières tendances qui se dégagent des scrutins de 2011 toujours en Egypte, qui indiquent une inversion des tendances et un recul de la catégorie « homme d'affaires » au Parlement ${ }^{69}$ ?

Que deviennent par ailleurs les réseaux de la clientèle «d'Etat », et notamment des cellules, comités de quartiers ou associations de partis tels que ceux du Rassemblement constitutionnel démocratique $(\mathrm{RCD})$ en Tunisie ${ }^{70}$ ? Certains ébauchent à ce sujet des hypothèses qu'il faudra désormais creuser; «Le RCD était l'acteur central de la politique clientéliste et redistributrice du régime autoritaire. Sa démarche était non seulement d'occuper le terrain de manière efficace mais aussi de relayer l'administration dans la mise en cuvre des politiques publiques. Du coup, le défunt RCD a donné naissance aujourd'hui à un groupe d'entremetteurs sociaux susceptibles d'exploiter le contexte d'incertitude politique et économique pour vendre au meilleur prix leur pouvoir d'intermédiation auprès des nouvelles organisations partisanes post-Ben Ali qui cherchent à attirer électoralement les populations désœuvrées des régions sinistrées. C'est dans ce contexte de nouvelle concurrence pour la médiation politique au local que des conflits, trop hâtivement qualifiés de 'tribaux' par la

\footnotetext{
${ }^{66}$ L. Zaki, 2009, « Pour une analyse localisée des élections marocaines... », article cité, p. 27.

${ }^{67}$ F. Mermier, S. Mervin (dir.), 2012, Leaders et partisans au Liban, Paris, Karthala/IFPO/IISMM ; M. Catusse, K. Karam, O. Lamloum (dir.), Métamorphose des figures du leadership au Liban. Champs et contrechamps des élections législatives de 2009, Beyrouth, Presses de l'Ifpo.

${ }^{68}$ S. Ben Néfissa, 2012, "Le temps électoral, le temps révolutionnaire et l'idiome islamiste en Egypte », in Institut de recherche sur la gouvernance, La gouvernance en révolution(s), Paris, Chroniques de la Gouvernance, ECLM.

${ }^{69}$ Voir à ce sujet l'article introductif de S. Ben Néfissa à ce volume.

${ }^{70}$ Sur le RCD, quelques études sur la décennie précédente : O. Feneyrol, 1997, «L'Etat à l'épreuve du local. Le réaménagement du quartier de 'Bab Souika-Halfaouine' à Tunis (1983-1992)», Monde arabeMaghreb/Machrek, n¹57, juillet-sept., pp. 58-68. M. Lahmar, A. Zghal, 1997, « La 'révolte du pain' et la crise du modèle de parti unique », in M. Ben Romdhane (dir.), Tunisie : mouvements sociaux et modernité, Dakar, Codestria, pp. 151-1992. Et plus largement M. Camau, V. Geisser, 2003, Le Syndrome autoritaire. Politique en Tunisie de Bourguiba à Ben Ali, Paris, Presses de Sciences Po ou B. Hibou, 2006, La force de l'obéissance. Economie politique de la répression en Tunisie, chap. 3, Paris, la découverte.
} 
presse, éclatent dans des zones paupérisées $d u$ Sud du pays ${ }^{71}$ ? La trajectoire surprise d'un Hachmi Hamdi et sa pétition populaire pour la liberté, la justice et le développement (alarîdha ech-cha'biyya) en Tunisie doit-elle, en ce sens, se comprendre entre autre comme l'exemple emblématique du développement de transactions matérielles sonnantes et trébuchante dans le cas particulier de la Tunisie, où le parti Ennahda ne disposait pas des mêmes réseaux d'action locale que les Frères musulmans égyptiens par exemple ? ou encore comme l'un des exemple de reconversion des anciens caciques du régime de Ben $\mathrm{Ali}^{72}$ ? En Egypte, les clivages et divergences du Parti national démocrate égyptien (PND) que l'on observait en particulier à l'échelle locale ${ }^{73}$ pourraient désormais donner quelques clés de compréhension des recompositions des rapports de forces?

Les valses-hésitations entre «politiques du nom » et «politique des partis », entre logiques personnelles et notabilières et dynamiques partisanes ${ }^{74}$ ont pris un nouveau tempo dans le double moment de la protestation d'abords, de l'élection ensuite. Les résultats des scrutins de 2011 et 2012 mettent en avant le savoir-faire électoral de formations partisanes telles que celles des Frères musulmans en Egypte et de Ennahda en Tunisie, à tel point que C. Steuer parle de "printemps des partis ${ }^{75}$, battant en brèche l'idée entretenue par le positionnement des acteurs protestataires eux-mêmes, qui exprimèrent souvent «un rejet populaire des partis politiques classiques» et mirent à distance les «partis de la place $»^{76}$. En ce sens, ils appellent à questionner la vie partisane telle qu'elle se réorganise avec le mouvement social. Certes, certaines des organisations installées n'ont rejoint les rangs de la révolution que prudemment ou tardivement; d'autres, les formations partisanes parlementaires marocaines par exemple, se sont explicitement démarquées de la protestation (elle-même encadrée par d'autres partis politiques, d'extrême gauche et islamistes), non sans clivages internes, notamment avec leurs jeunesses; mais là encore les nuances s'imposent compte tenu de la diversité des situations. Les cadres, dirigeants et militants des partis de l'opposition «historique » à ces régimes, essentiellement de gauche radicale et islamistes se trouvent confrontés à l'opportunité mais aussi l'épreuve du passage de l'illégalité à la légalité, pour

71 A. Allal, 2011, «Tunisie: le bal des prétendants », archives du CERI, http://www.ceri-sciencespo.org/archive/2011/octobre/chro_aa.pdf

${ }^{72}$ Ce n'est pas la lecture qu'en propose M. Kerrou par exemple, qui s'interroge plus volontiers sur l'épaisseur anthropologique de ce vote et la mobilisation de réseaux confrériques et tribaux. Voir M. Kerrou, 2011, «L'énigme d'al-Arîdha ech-cha 'biyya (pétition populaire pour la liberté, la justice et le développement) », intervention à la Journée d'étude «Processus électoraux, révolutions et transitions en Egypte et en Tunisie », Tunis, IRMC/IRD/IREMAM.

73 Voir D. Al-Khawaga 1997, «Le Parti national démocrates et les élections de 1995 : la conjonction de nombreuses logiques d'action », in S. Gamblin (dir.), Contours et détours..., op. cit., pp. 75-92 ; L. Blaydes, S. el Tarouty, 2011, « La concurrence interne au Parti national démocrate égyptien », in F. Kohstall, F. Vairel (dir.), «Fabrique des élections... », op. cit, pp. 69-93.

${ }^{74}$ Voir au Maroc, M. Tozy (dir.), 2010, Élections au Maroc : Entre partis et notables (2007-2009), Casablanca, Centre marocain des sciences sociales/Fondation Konrad Adenauer.

75 C. Steueur, 2011, «Le printemps des partis ? Le rôle des organisations partisanes dan la mobilisation électorale », intervention à la Journée d'étude « Processus électoraux, révolutions et transitions en Egypte et en Tunisie », Tunis, IRMC/IRD/IREMAM.

${ }^{76}$ V. Geisser, 2012, « Les protestations populaires à l'assaut des régimes autoritaires : une 'révolution' pour les sciences sociales ? », Année du Maghreb 2012, introduction au dossier de recherche, p. 14. 
certains de l'exil au retour au pays, de l'action radicale à la formation de coalition de gouvernement.

D'autres points attirent l'attention quand aux recompositions des scènes partisanes : quid de l'empreinte laissée par l'ancien régime sur les anciens partis d'opposition, décrits dans le cas de la Tunisie pour beaucoup comme des "proto-partis ${ }^{77}$, fonctionnant au service d'une personnalité, "dépourvus de base sociale et déconnectés du mouvement social qui a entraîné la fuite de Ben Ali ${ }^{78}$, des «organisations politiques n'exist[ant] que par et pour l'autoritarisme » dont on pouvait penser qu'elles «perd[raient] toute vocation politique avec [la] disparition de [celui-ci] ${ }^{79}$ ? Comment se joue à l'échelle locale notamment l'emprise croissante de la compétition politique par des organisations partisanes ${ }^{80}$ et par les notabilités ancrées, alors même que ces dernières furent nettement moins présentes dans le moment protestataire? Comment analyser aussi et surtout le savoir faire électoral de ces partis «islamistes » singulièrement efficaces dans la politique électorale ? A la lumière des questions posées plus haut, ce dernier point soulève plusieurs problèmes de comparaison. Un le constat se répète de contextes en contextes : les partis islamistes s'avèrent de remarquables machines électorales du Maroc à la Turquie, en passant par le Liban, la Palestine, l'Algérie, et aujourd'hui la Tunisie et l'Egypte ${ }^{81}$. Mais à l'observation des scrutins de 2011, des divergences se dessinent aussi qu'il faudra questionner sans céder au piège des ressemblances : les Frères musulmans disposent d'un solide réseau d'entraide et de services en Egypte, ce n'est pas en revanche le cas en Tunisie, où pour le coup l'hypothèse d'une traduction en voix de relations de clientèle de longue date est nettement moins évidente; de même les Frères musulmans égyptiens pouvaient être considérés comme des outsiders du champs politique du point de vue du droit qui leur interdisait formellement l'accès au jeu jusqu'en 2011, mais ils en étaient aussi des insiders expérimentés, qui non seulement mobilisaient, travaillaient sur «dans la rue », avec des répertoires d'action plastiques, et ont participé à la plupart des scrutins nationaux depuis les années 1980, de manière « contournée ${ }^{82}$. Ceci n'est pas le cas de la coalition-parti «salafiste » égyptienne Al-Nour, constituée au lendemain de la révolution autour de l'organisation de la $d a$ ' $w a$, dont les scores sont remarquables, ni d'Ennahda en Tunisie qui ne peut non plus se prévaloir d'expériences régulières de campagne électorale, et figuraient a priori plus comme des organisations plus «novices » qu'expérimentées en termes d'organisation de scrutin. A la différence du Parti de la justice et du développement (PJD) marocain qui emporte également les législatives de novembre 2011 ou de l'Adalet ve Kalkınma Partisi (AKP) Turc qui dirige le pays depuis

\footnotetext{
${ }^{77}$ M. Camau, V. Geisser, 2003, Le syndrome autoritaire..., op. cit., pp. 237 et ss.

${ }^{78}$ A. Allal, 2011, « Tunisie : le bal des prétendants », article cité, p. 1.

${ }^{79}$ M. Camau, V. Geisser, 2003, Le syndrome autoritaire ..., op. cit., pp. 237.

${ }^{80}$ Certains évoquent la «partisanisation» de la vie politique dans ces moments de changements de régime : J. Heurtaux, 2003, « L'emprise des partis sur la compétition politique en Pologne : les candidats face à la partisanisation (1990-2000) », in A. Roger (dir.), Des partis pour quoi faire ? La représentation politique en Europe centrale et orientale, Bruxelles, Bruylant, pp. 77-101.

81 A l'échelle des municipalités, voir A. Signoles (dir.), «Dossier: les municipalités islamistes », Critique internationale, vol. $42, \mathrm{n}^{\circ} 1$.

${ }^{82}$ M. Vannetzel, 2008, « Les voies silencieuses de la contestation... », article cité.
} 
2002, ni les Frères musulmans égyptiens, ni al-Nour, ni Ennahda n'ont pu en outre s'appuyer dans leurs conquêtes des sièges parlementaires sur des expériences de scrutins locaux préalables.

En somme donc, les chantiers sont ouverts pour étudier les recompositions des leaderships politiques à la faveur de la révolution et de ces élections exceptionnelles : celles-ci enregistrent bien sûr la délinquance de structures de mobilisation, à commencer par celles des machines partisanes de l'ancien régime, l'émergence de nouveaux leaderships (dont il faudra questionner leurs cristallisations dans le mouvement social ${ }^{83}$ ) ou encore la réorganisation d'anciennes forces politiques qui passent de l'opposition illégale vers des formes pluralistes et régulières de compétitions politiques. Mais ces élections sont également elles-mêmes productrices de rapports de forces et d'une ingénierie politique qui favorisent les uns et marginalisent les autres. Cela demande en tout état de cause de questionner sur le long terme les continuités biographiques et leurs infléchissements, les logiques de mobilisation et de démobilisation qui s'observent dans les séries mouvement social / révolution / processus électoraux, ou encore, l'évolution des foyers de socialisation politique et le poids des expériences générationnelles.

\section{Conclusion : une sociologie du comportement de l'électeur-rice égyptien-ne et tunisien- ne}

$\mathrm{Au}$ final, la rupture essentielle produite par la révolution sur nos grilles de lectures est la découverte de l'électeur. Une découverte à deux niveaux au moins : l'un théorique, accorde soudain une importance aux votes et préférences des électeurs, en considérant que les conditions sont réunies désormais pour accorder un crédit sociologique aux résultats des élections; l'autre empirique et méthodologique, concerne l'accès aux données et à la possibilité matérielle de se livrer enfin à une sociologie électorale telle qu'on a pu la conduire ailleurs. Ces travaux n'en sont qu'à leurs prémices mais l'enquête menée en Tunisie par A. Gana et G. Van Hamme ${ }^{84}$ montre toutes les possibilités que cela peut ouvrir. En particulier, la sociologie du vote devrait contribuer à penser les accrochages et décrochages entre le moment protestataire et le moment électoral. Plutôt que de partir de l'idée d'une confiscation de la révolution des uns par les machines électorales des autres, il s'agit au contraire de comparer différents modes d'expressions du pluralisme, le vote pas plus que la manifestation finalement ne pouvant être saisis comme les «seules formes recevables d'expression des demandes sociales ${ }^{85}$. Certains opposeront le travail de $«$ professionnels » de la politique, plus ou moins spécialistes de la joute politique (plus ou moins car les régimes jusqu'à présent ont justement entravé les conditions de la compétition électorale) à l'engagement des «profanes » que

\footnotetext{
${ }^{83}$ S. Soliman, 2011, « Certains ont quand même réussi à se faire élire ! Analyse de la performance électorale des forces 'civiles' en Egypte », intervention à la Journée d'étude « Processus électoraux, révolutions et transitions en Egypte et en Tunisie », Tunis, IRMC/IRD/IREMAM.

${ }^{84}$ Voir la contribution de A. Gana à ce volume.

${ }^{85}$ S. Deschezelles, S. Luck, 2011, op. cit., p. 9.
} 
seraient ces «inconnus » outsiders mobilisés pour faire chuter le régime ${ }^{86}$. Mais ici il s'agit plutôt de comparer différents rapport au champ politique tels qu'ils se sont déclinés dans les comportements des manifestants (dans les faits, une infime part des populations tunisiennes et égyptiennes), les votants, les abstentionnistes, etc. On attend désormais les croisements de la sociologie électorale qui point avec celle des mouvements révolutionnaires qui elle aussi commence à se dessiner ${ }^{87}$ : en quoi le moment insurrectionnel est-il lui-même un moment d'ordonnancement et d'encadrement du paysage politique, où se jouent d'âpres discussions sur les (nouvelles) règles du jeu, mais aussi la disparition, l'émergence ou la reconversion de « représentants », de «porte-paroles », de « leaderships », ou encore des formes de solidarités singulières. Entre autres questions qui se dégagent de ces séquences rapides des histoires politiques tunisiennes et égyptiennes, les logiques variées du vote (ou de l'abstention) des «pauvres»- et en retour celle du vote des «bourgeois» ou des «classes moyennes » en contexte de fortes incertitudes sur les performances économiques des nouveaux régimes; la réinvention de solidarités «primordiales », quelles soient communautaires, confessionnelles ou tribales ${ }^{88}$; les effets comparés des inégalités voire frustrations relatives perçues dans le mouvement social, à questionner dans les superpositions de cartes de la contestation et celles du vote par exemple qui produiront très certainement des dissemblances en Tunisie et en Egypte; ou encore le devenir des liens clientélistes et l'expression d'identités ou de préférences partisanes en fonction de trajectoires biographiques et de variables socioéconomiques.

\footnotetext{
${ }^{86} \mathrm{Ph}$. Corcuff, L. Mathieu, 2009, «Partis et mouvements sociaux : des illusions de l'actualité à une mise en perspective sociologique », Actuel Marx, n46, pp. 67-80.

87 Voir entre autre M. Bennani-Chraïbi, O. Fillieule (dir.), à paraître, «Révoltes et révolutions dans le monde arabe [titre provisoire] », Revue français de science politique, vol. 62 ; A. Allal, T. Pierret (dir.), à paraître, Au cour des révoltes arabes. Portée des processus révolutionnaire [titre provisoire], Paris, Karthala/Iremam; V. Geisser (dir.), à paraître, «Les protestations populaires à l'assaut des régimes autoritaires : une 'révolution' pour les sciences sociales ? », L'Année du Maghreb 2012, Dossier de recherche, vol. VIII.

${ }^{88}$ A titre de comparaison: M. Hachemaoui, 2012, «Y-a-t-il des tribus dans l'urne ? Sociologie d'une énigme électorale (Algérie) », Cahiers d'études africaines mars, n²05, pp. 103-163.
} 